\title{
Three-dimensional skyrmion states in thin films of cubic helimagnets
}

\author{
F. N. Rybakov ${ }^{1,2}{ }^{*}$ A. B. Borisov ${ }^{1}$, and A. N. Bogdanov ${ }^{2 \dagger}$ \\ ${ }^{1}$ Institute of Metal Physics, Ekaterinburg, 620990, Russia and \\ ${ }^{2}$ IFW Dresden, Postfach 270116, D-01171 Dresden, Germany
}

(Dated: September 20, 2012)

\begin{abstract}
A direct three-dimensional minimization of the standard energy functional shows that in thin films of cubic helimagnets chiral skyrmions are modulated along three spatial directions. The structure of such 3D skyrmions can be thought of as a superposition of conical modulations along the skyrmion axis and double-twist rotation in the perpendicular plane. Numerical solutions for chiral modulations demonstrate that 3D skyrmion lattices and helicoids are thermodynamically stable in a broad range of applied magnetic fields. Our results disclose a basic physical mechanism underlying the formation of skyrmion states recently observed in nanolayers of cubic helimagnets.
\end{abstract}

The Dzyaloshinskii-Moriya (DM) interactions arising in noncentrosymmetric magnets as a result of their crystallographic handedness, induce long-range modulations with a fixed sense of the magnetization rotation [1]. It was also established that DM interactions provide a unique mechanism to stabilize two- and three-dimensional (2D and 3D) localized states (chiral skyrmions and hopfions) and their bound states (skyrmion lattices) [2-4].

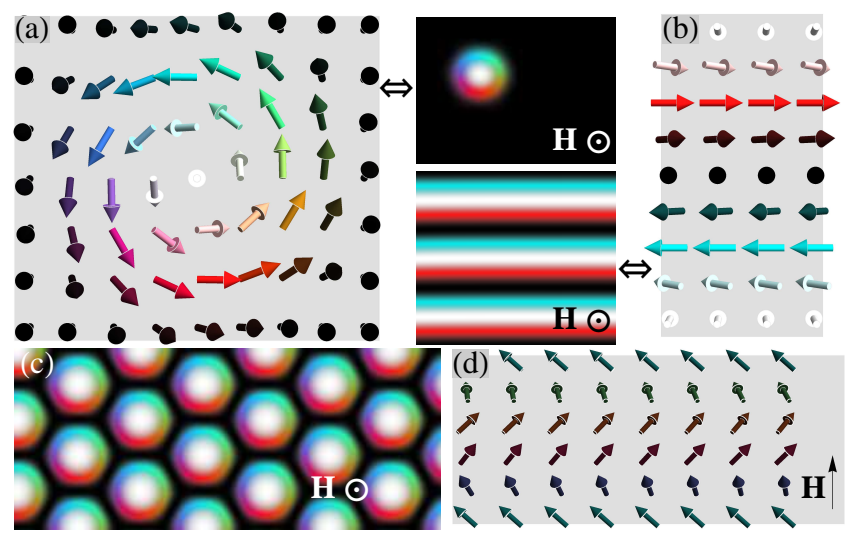

Figure 1: (color online). Magnetic configurations in basic chiral modulations arising in cubic helimagnets: (a) an isolated skyrmion with double-twist modulations perpendicular to the applied field, (b) transversally distorted helices (helicoids), (c) a hexagonal lattices of chiral skyrmions (a), (d) longitudinal distorted helices (cones).

Skyrmion lattices and isolated skyrmions have been recently discovered in nanolayers of cubic helimagnets [58] and in monolayers of magnetic metals with induced DM interactions [9]. Chiral skyrmions are highly mobile nanoscale spots of reverse magnetization that can be utilized in ultra dense recording technologies and in the emerging spin electronics $[6,10]$. Importantly, in the majority of nonlinear field models static multidimensional

\footnotetext{
* Corresponding author: f.n.rybakov@gmail.com

† a.bogdanov@ifw-dresden.de
}

solitons are unstable and collapse spontaneously into topological singularities [11]. This singles out condensedmatter systems with broken chiral symmetry as a special class of materials, where chiral skyrmions can be formed as static, mesoscopic solitons and can be manipulated in broad ranges of the thermodynamical parameters [2, 3].

The experimental observations of skyrmions and other chiral modulations $[5,7,8,12]$ are in an excellent agreement with results of the phenomenological theory based on the classical Dzyaloshinskii model [1, 3, 12, 13]. Particularly, theoretical analysis shows that induced uniaxial anisotropy stabilizes skyrmions and helicoids in thin layers of cubic helimagnets $[12,14]$. In bulk cubic helimagnets, these chiral modulations exist as metastable states $[3,14]$. Recent experiments in MnSi and FeGe confirm that they are stabilize by an induced uniaxial anisotropy $[7,8,12]$. On the other hand, nothing is known about a role of induced magnetic anisotropy in mechanically thinned nanolayers of cubic helimagnets where direct observations of skyrionic states have been reported $[5,6]$. Generally, the exact magnetic structures of skyrmions and other modulated states arising in confined cubic helimagnets are still not resolved and physical mechanisms underlying their formation and stability are not completely understood.

In this paper we provide the first three-dimensional calculations of chiral modulated states in thin layers of cubic helimagnet films. We show that below a critical thickness, a double-twist skyrmion gains additional modulations along its axis. The calculated phase diagram for $3 \mathrm{D}$ skyrmions and competing phases indicates a broad range of the films thickness, where skyrmionic states are thermodynamically stable.

Within the phenomenological theory introduced by Dzyaloshinskii [1], the magnetic energy density of a cubic non-centrosymmetric ferromagnet can be written as $[1,15]$

$$
w=A(\operatorname{grad} \mathbf{m})^{2}+D \mathbf{m} \cdot \operatorname{rot} \mathbf{m}-\mathbf{H} \cdot \mathbf{m} M .
$$

$\mathbf{m}=(\sin \theta \cos \psi ; \sin \theta \sin \psi ; \cos \theta)$ is the unity vector along the magnetization $\mathbf{M}$ and $\mathbf{H}$ is the applied field. Energy density functional (1) includes only the principal interactions essential to stabilize skyrmions and helicoids: 
the exchange stiffness with constant $A, \mathrm{DM}$ coupling energy with constant $D$, and the Zeeman energy. We neglect less important energy contributions such as intrinsic and induced magnetic anisotropies or stray-fields. In contrast to common magnetically soft materials where magneto-dipole interactions play an important role [16], stray-field effects in noncentrosymmetric magnets are found to be weak due to the stabilizing influence of the DM interactions [10].

DM interactions favour spatial modulations of $\mathbf{M}$ with a fixed rotation sense [1]. In an infinite sample, isolated skyrmions (Fig. 1 a) are described by solutions of type $[2,3]$,

$$
\theta=\theta(\rho), \quad \psi=\varphi+\pi / 2 .
$$

Here we write the spatial variables in the terms of cylindrical coordinates $r=(\rho \cos \varphi ; \rho \sin \varphi ; z)$. Eq. (2) describes $2 \mathrm{D}$ skyrmions as axisymmetric tubes with "double-twist" modulations in the $(x, y)$ plane and a homogeneous distribution along the skyrmion axis ( $z$-axis) (Fig. 1 a) [3, 17]. The solutions for one-dimensional modulations include distorted helices with the propagation direction perpendicular to the applied field, helicoids (Fig. 1 b), and longitudinal distorted helices modulated along the field, cones (Fig. $1 \mathrm{~d}$ ). The analytical solutions for helicoids describe a gradual unwinding of chiral coils in an increasing magnetic field [1]. The equilibrium parameters for the cone phase are given by [15]

$$
\cos \theta=|\mathbf{H}| / H_{D}, \quad \psi(z)=\psi_{\text {cone }}(z) \equiv 2 \pi z / L_{D},
$$

where the period of the helix, $L_{D}$ and the saturation field, $H_{D}$ are expressed as

$$
L_{D}=4 \pi A /|D|, \quad H_{D}=D^{2} /(2 A M) .
$$

We introduce the reduced energy density of a modulate phase as $e=\langle w\rangle /\left[D^{2} /(4 A)\right]$ where $\langle w\rangle$ is the energy density averaged over the modulation period. Then the equilibrium energy density of the cone phase can be written as

$$
e_{\mathrm{cone}}=-\left[1+\left(H / H_{D}\right)^{2}\right]
$$

In an infinite sample, the cone (3) corresponds to the global minimum of model (1) in the entire magnetic field range below the saturation field $H_{D}(4)$. Helicoids and skyrmion lattices only exist as metastable states [14].

Contrary to lower symmetry noncentrosymmetric ferromagnets [2], in cubic helimagnets DM interactions provide three equivalent modulation directions. However, $2 \mathrm{D}$ chiral skyrmions investigated in $[3,13]$ have only two (in-plane) modulation directions while along the skyrmion axis the structure remains homogeneous. Thus, the question arises: is it possible to lower the skyrmion energy by imposing additional chiral modulations along the skyrmion axis? Our calculations based on rigorous solutions of micromagnetic equations show that the formation of 3D skyrmions actually occurs in the thickness range below a critical thickness.
As a model we consider a film of thickness $L$, infinite in $x$ and $y$ directions and bounded by parallel planes at $z= \pm L / 2$. Numerical solutions for chiral modulations are derived by direct minimization of functional (1) with free boundary conditions along the $z$-axis and periodic boundary conditions in the $(x, y)$ plane. This yields the equilibrium spatially inhomogeneous distributions of the magnetization vector $\mathbf{m}$ in the layer as functions of the three spatial variables and the two control parameters, reduced magnetic field, $H / H_{D}$ and confinement ratio, $L / L_{D}$

A three-dimensional minimization of nonlinear sigma models needs huge computing efforts and is usually executed on high-performance supercomputers [18]. Modern graphics cards offer a promising alternative. However, this method requires special hardware-oriented highly parallel algorithms [19]. We have developed a special algorithm for CUDA architecture for NVIDIA graphics cards. This architecture has been found to be efficient to solve complex micromagnetic problems [20]. We use a nonlinear conjugate gradient method to minimize energy functional (1). The results of the minimization have been checked by the compatibility with the EulerLagrange equations. The results of numerical minimization of model (1) are presented in Figs. 2, 3, 4 and as video [21].
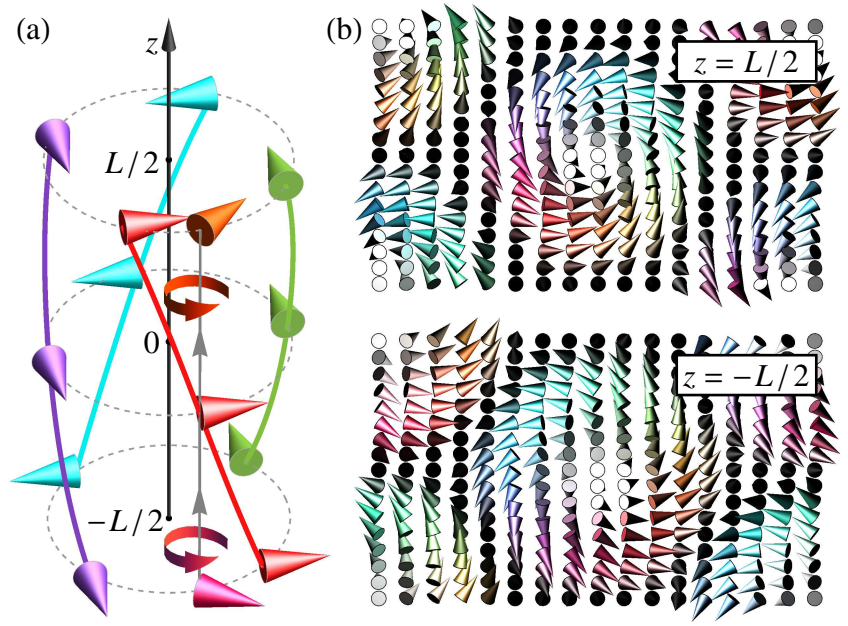

Figure 2: (color online). 3D skyrmion lattice: (a) Distribution of the magnetization in the skyrmion core demonstrates chiral conical modulations along the cell axis. For clarity, the sizes along the $z$-axis are magnified; (b) Calculated distribution of the magnetization in the skyrmion cell for top and bottom layers in a film with thickness $L / L_{D}=0.25$ and in the applied field $H / H_{D}=0.2$.

Isolated and embedded skyrmions. The equilibrium magnetic configurations in a 3D skyrmion lattice strongly differ from axisymmeteric $2 \mathrm{D}$ skyrmions arising in infinite helimagnets (2). The magnetization vector $\mathbf{m}$ depends on all three spatial components, $(\rho, \varphi, z)$, and exhibits complex three-dimensional modulations ((Fig. 2) and [21]). 
It was, however, found that a simplified ansatz

$$
\theta=\theta(\rho), \quad \psi(\phi, z)=\varphi+\pi / 2+\widetilde{\psi}(z)
$$

with $\widetilde{\psi}(z)=\psi_{\text {cone }}(3)$ provides a nice approximation of the solutions for isolated and bound 3D skyrmions.

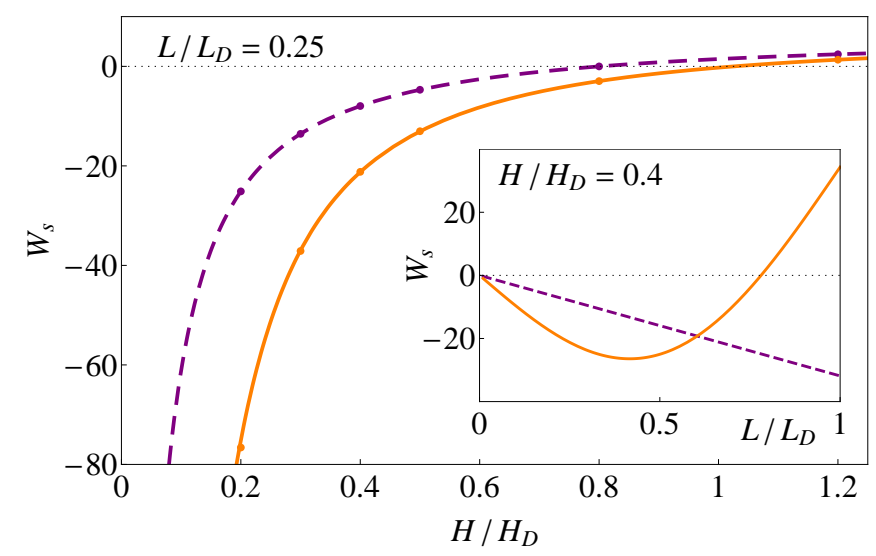

Figure 3: (color online). Calculated equilibrium energies of isolated skyrmions with ansatz (6) (solid line) and for those that are homogeneous along the $z$-axis (2D skyrmions, dashed line) as functions of the reduced field $H / H_{D}$ in a film with reduced thickness $L / L_{D}=0.25$. Inset shows the equilibrium energies of the skyrmions as functions of the reduced thickness at a fixed applied field

$$
H / H_{D}=0.4 \text {. }
$$

Eq. (6) describes double-twist rotations in the $(x, y)$ plane and conical modulations (3) along the skyrmion axis (Fig. 2 a). Remarkably, the period of conical modulations in the skyrmion coincides with the helix period, $L_{D}$. We introduce the reduced equilibrium energy of an isolated skyrmion as

$$
W_{s}=\frac{1}{\left(A L_{D}\right)} \int_{0}^{2 \pi} d \varphi \int_{-L / 2}^{L / 2} d z \int_{0}^{\infty}\left(w-w_{0}\right) \rho d \rho
$$

where $w_{0}=-H M$ is the energy density of the saturated state. In Fig. 3 The energies $W_{s}$ for isolated skyrmions with ansatz (6) and for solutions homogeneous along the skyrmion axis (2D skyrmions) are plotted as functions of the applied field in Fig. 3 (main figure) and the reduced film thickness (inset). These results show that in a broad range of the control parameters $H / H_{D}$ and $L / L_{D}$, the modulation along the skyrmion axis reduces its energy. 3D modulated skyrmions should not be confused with three-dimensional topological chiral solitons (hopfions) [4].

Helicoids and cones. In thin films, helicoids become inhomogeneous along the film thickness [21]. However, we failed to find a simple ansatz to describe these distortions. Conical states propagating perpendicular to the layer surfaces are compatible with the film geometry, and the free boundary conditions impose no constrictions on these modulations. As a result, the solutions for the cone phase in the film and the equilibrium energy density equal to those in a bulk sample (3), (5) [14, 15]. Conical states (3) can exist even in films with thickness smaller than the helix period $\left(L<L_{D}\right)(4)$. In this case $\Delta \psi=|\psi(L / 2)-\psi(-L / 2)|=2 \pi L / L_{D}<2 \pi$.

The calculated equilibrium energy densities of helicoids $\left(e_{\mathrm{h}}\right)$ and 3D skyrmion lattices $\left(e_{\mathrm{sk}}\right)$ have been compared with the cone energy density $\left(e_{\text {cone }}\right)$. The results are plotted as functions $\Delta e_{\mathrm{h}}=\left(e_{\mathrm{h}}-e_{\text {cone }}\right)$ and $\Delta e_{\mathrm{sk}}=\left(e_{\mathrm{sk}}-e_{\mathrm{cone}}\right)$ versus the applied field together with the corresponding results for $1 \mathrm{D}$ helicoids and 2D skyrmion lattices arising in bulk cubic helimagnets $[1,3]$ (Fig. 4 a). In the latter case the cone has the lowest energy in the entire region below the saturation field, $H_{D}$ (3). This situation retains in sufficiently thick films (see Fig. 3 Inset). However, the energy balance between the chiral phases drastically changes in thin layers below the critical thickness. Here the formation of 3D modulations is resulted in a large reduction of the helicoids and skyrmion lattices and leads to their energetic advantage over the cone phase. (Fig. 4 (a)). It means that in sufficiently thin cubic helimagnets films the onset of conical modulations in helicoids and skyrmion lattices provides a specific mechanism to stabilize these chiral modulations in a broad range of applied magnetic fields (Fig. 4).
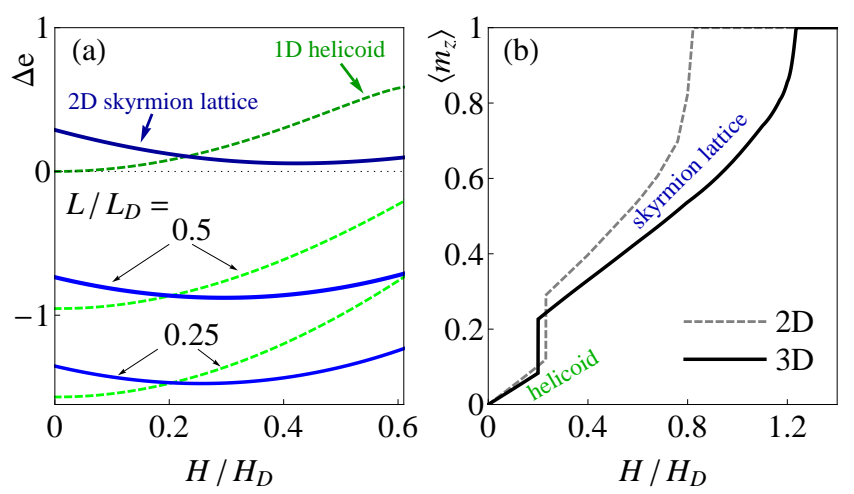

Figure 4: (color online). (a) The differences $\Delta e$ between the energies of skyrmion lattice and the cone phase (solid, blue) and between the helicoid and the cone phase (dashed, green) as functions of the applied field for 2D and $3 \mathrm{D}$ modulations. Functions $\Delta e\left(H / H_{D}\right)$ are plotted for two values of reduced film thicknesses $L / L_{D}=0.25$ and 0.5 in comparison with results derived for $1 \mathrm{D}$ helicoids [1] and hexagonal skyrmion lattices homogeneous along the skyrmion axis (2D skyrmions investigated in [3]). (b) Magnetization curves for 3D modulated structures in a film with reduced thickness $L / L_{D}=0.25$ (solid lines) in comparison with the magnetization curves for 1D helicoids and 2D skyrmion lattices [1, 3].

An elementary analysis of modulations (6) allows to understand basic physical mechanisms underlying the formation of 3D skyrmions. 
Inserting ansatz (6) into (1) yields the following expression

$$
\begin{aligned}
\tilde{w} & =A\left(\theta_{\rho}^{2}+\frac{\sin ^{2} \theta}{\rho^{2}}\right)+\underbrace{D \cos \widetilde{\psi}(z)}_{\widetilde{D}(z)}\left(\theta_{\rho}+\frac{\sin 2 \theta}{2 \rho}\right) \\
& -H M \cos \theta+\underbrace{\sin ^{2} \theta\left[A \widetilde{\psi}_{z}^{2}(z)-D \widetilde{\psi}_{z}(z)\right]}_{w_{z}} .
\end{aligned}
$$

In functional (8) three first terms are responsible for the formation of double-twist modulations in the $(x, y)$ plane [3] and $w_{z}(z)$ describes modulations along the skyrmion axis. With conical modes $\widetilde{\psi}(z)=\psi_{\text {cone }}(3)$, the function $\widetilde{D}(z)$ and energy density $w_{z}$ can be written as

$$
\widetilde{D}(z)=D \cos \left(2 \pi z / L_{D}\right), \quad w_{z}=-D^{2} \sin ^{2} \theta /(4 A) .(9)
$$

Energy density (8) includes two different DM terms. The first, with factor $\widetilde{D}(z)$, favours double-twist rotations in the $(x, y)$ plane, the other induces cone-mode modulations along the $z$-axis. The energy gain from the double-twist modulations $\propto \widetilde{D}^{2}(z)$ reaches the maximum at $\widetilde{\psi}(z)=0$. The cone modulations increase the double-twist energy. On the other hand, these yield a negative energy contribution $w_{z}(9)$ into the total energy. The equilibrium 3D skyrmion patterns are formed as a result of the competition between different DM interaction terms.

In the calculated 3D skyrmion textures angle $\widetilde{\psi}$ equals zero in the layer center and rotates with a same rotation sense to the maximal value at the surfaces, $|\widetilde{\psi}( \pm L / 2)|=$ $\pi L / L_{D}$. Importantly, for $\widetilde{\psi}>\pi / 2$ the factor $\widetilde{D}(z)$ becomes negative. It means that cubic helimagnets films with thickness larger than $L_{D} / 2$ include regions where function $\widetilde{D}(z)$ becomes negative corresponding to the double-twist modes with energetically unfavorable sense of rotation. This explains the existence of the critical thickness for 3D modulations and allows to estimate its value. As a rule of a thumb, the critical thickness can be estimated as a half of the helix period $L_{D}$ (see e.g. Fig. 3 Inset).

The first direct observations of chiral skyrmions have been reported in a mechanically thinned cubic helimagnet $(\mathrm{Fe}, \mathrm{Co}) \mathrm{Si}$ with thickness $L=20 \mathrm{~nm}$ [5]. In this material $L_{D}=90 \mathrm{~nm}$, and, thus, confinement ratio $L / L_{D}=0.22$. This is quite below the critical thickness, and ,thus, 3D modulations are expected to exist in this film. In Fig 4 (b) we present the calculated magnetization curve for a film with confinement ratio $L / L_{D}=0.25$ close to that of the film investigated by $\mathrm{Yu}$ et al. [5]. This indicates the stability regions for helicoids and skyrmions, the first order transitions between helicoid and skyrmion lattices, and the second order transition of the skyrmion lattice into the saturated state.

Earlier an alternative mechanism to stabilize chiral skyrmions in cubic helimagnets has been proposed by Butenko et al. [14]. According to this paper the distortions of a cone phase imposed by uniaxial magnetic anisotropy strongly increase its energy. As a result, chiral skyrmion lattices and helicoid become thermodynamically stable in a broad range of applied magnetic fields (Fig. 3 in [14]). In our recent studies, the cone modes remain undistorted. The energetic advantage of 3D skyrmion lattices and helicoids is gained because 3D modulations grant a larger reduction of the DM energy than single-direction modulations in cone modes. New experiments are needed for detailed investigations of induced magnetic anisotropy and to resolve the exact magnetic structures in confined cubic helimagnets. These will allow to understand a role of unaxial distortions and 3D modulations in the formation of skyrmion states in these systems.

In conclusion, numerically calculated 3D solutions for chiral modulations in nanolayers of cubic heliimagnets show that in the films below the critical thickness (estimated as a half of the helix period) chiral skyrmions and helicoids are sufficiently inhomogeneous along three spatial directions. Such 3D modulated skyrmions and helicoids modulated along the layer thickness are thermodynamically stable in a broad range of applied magnetic fields.

We thank T. Monchesky for critically reading the manuscript and fruitful advice.
[1] I. E. Dzyaloshinskii, Sov. Phys. JETP 19, 960 (1964), 20, 665 (1964).

[2] A. N. Bogdanov and D. A. Yablonsky, Zh. Eksp. Teor. Fiz. 95, 178 (1989) [Sov. Phys. JETP 68, 101 (1989)]; A. Bogdanov, JETP Lett. 62, 247 (1995).

[3] A. Bogdanov and A. Hubert, J. Magn. Magn. Mater. 138, 255 (1994), 195, 182 (1999); A. N. Bogdanov et al., Physica B 359-361, 1162 (2005).

[4] A. B. Borisov, F. N. Rybakov, Low Temp. Phys. 36, 766 (2010).
[5] X. Z. Yu et al. Nature, 465, 901 (2010); A. Tonomura et al., Nano Lett. 12, 1673 (2012); S. Seki et al., Science 336, 198 (2012).

[6] X. Z. Yu et al., Nat. Mater. 10, 106 (2011); X. Z. Yu et al., Nat. Commun. 3, 988 (2012).

[7] S. X. Huang and C. L. Chien, Phys. Rev. Lett. 108, 267201 (2012).

[8] M. N. Wilson et al., Phys. Rev. B 86, 144420 (2012).

[9] S. Heinze et al., Nature Phys. 7, 713 (2011).

[10] N. S. Kiselev et al., J. Phys. D: Appl. Phys. 44, 392001 (2011). 
[11] G. H. Derrick, J. Math. Phys. 5, 1252 (1964).

[12] E. A. Karhu et al., Phys. Rev. B 85, 094429 (2012).

[13] U. K. Rößler et al., J. Phys.: Conf. Ser. 303, 012105 (2011).

[14] A. B. Butenko et al., Phys. Rev. B 82, 052403 (2010).

[15] P. Bak and M. H. Jensen, J. Phys. C: Solid State Phys. 13, L881 (1980).

[16] A. Hubert, R. Schäfer, Magnetic Domains (Springer, Berlin, 1998).
[17] G. H. Wright and N. D. Mermin, Rev. Mod. Phys. 61, 385 (1989).

[18] J. Jäykkä and J. Hietarinta, Phys. Rev. D 79, 125027 (2009).

[19] D. B. Kirk and W. W. Hwu, Programming Massively Parallel Processors (Elsevier, Amsterdam, 2010).

[20] A. Vansteenkiste, B. Van de Wiele, J. Magn. Magn. Mater. 323, 2585 (2011).

[21] See www.youtube.com/user/helimagnets for a demonstration of the magnetic structures of the cone, helicoid, and skyrmion phases in a thin film. 